\title{
LA TEORIA DE LAS PREGUNTAS, LOS PODERES EPISTEMICOS Y LA TEORÍA INDEXICA DEL CONOCIMIENTO (II)
}

16. Una dimensión de la semántica de 'saber' y 'conocimiento': la teoria de las preguntas y las guisas proposicionales. Debemos generalizar sobre el ejemplo de Powers. Tanto Platón como Powers parten de una pregunta acerca de $q u \dot{e}$ es el caso como $(Q),{ }^{*}$ en lugar de partir de una pregunta acerca de si algo es el caso. Indudablemente existen muchas otras preguntas: dónde, cuándo, cómo, por qué, con qué, de dónde, etc. Todas estas preguntas pueden hacerse con los mismos recursos de la oración que formula la respuesta correcta. Todas son preguntas que seleccionan un componente de la respuesta correcta como el punto de interrogación, por así decirlo. Pero tales preguntas no agotan los puntos de interrogación de una proposición. Por ejemplo, podemos preguntar por las conexiones lógicas entre dos proposiciones, o funciones proposicionales, cuando éstas forman una proposición compuesta o una función proposicional. Sin embargo, el lenguaje ordinario no tiene mecanismos sencillos que nos permitan plantear tales preguntas sin describir Ia proposición generadora de la pregunta en lugar de presentarla simplemente.

Considérese la proposición expresada por esta oración:

2) Si todos los vuelos fueran puntuales hoy, Juan está en la sala de espera o en una caseta telefónica, o bien, no vino.

Lógicamente, aun si no prácticamente, podriamos plantear preguntas que tuvieran la conectiva principal 'si...' como el punto de interrogación, o la disyunción 'o' del consecuente, o el cuantificador universal 'todos' del antecedente, o el tiempo denotado por la expresión 'puntual', etc. En el caso de la oración (2) parece que cada palabra, expresión y cláusula determinia un punto de interrogación. Esto incluye la agrupación de preguntas tales como "Si todos los vuelos fueran puntuales hoy, ¿dónde está Juan?", y la pregunta general: "¿Es el caso que (2)?"

Quizá podamos reunir toda la familia de preguntas de la siguiente manera:

a) Tenemos la jerarquía de las formas lógicas de una proposición que es la respuesta correcta para cada miembro de una familia de preguntas correspondiente; por ejemplo, en el caso de (2) tenemos las formas: $p$ (de una proposición cualquiera), $p \supset q$ (de algún condicional cualquie-

* Véase la sección 15 en la primera parte de este artículo, publicada en Diánoia 1979. 
ra), $p \supset(q \vee r)$ (para cualquier condicional con una disyunción como consecuente), $(\forall x)(F x \supset q \vee r)$ (de un condicional con un antecedente universal y un consecuente disyuntivo), etc. Además tenemos las formas más y más profundas de (2), por ejemplo, las que resultan del análisis de cualesquiera de sus conceptos componentes. Si analizamos vuelo, sala de espera, caseta telefónica, venir, etc., encontramos una forma lógica muy compleja por debajo de la forma ostensible que revela la oración (2). ${ }^{24}$

b) Tomamos cada componente o parte de una proposición considerada como de una cierta forma lógica, como un punto de interrogación, ya sea que la parte sea una parte propiamente o no. Si analizamos cualquier componente de (2), por ejemplo, 'vuelo' o 'teléfono', encontramos más puntos de interrogación.

EI resultado es una jerarquía de la familia de preguntas, determinada por una proposición dada. Cada uno de los niveles de preguntas corresponde a lo que en [12], parte II, he llamado una guisa proposicional. Una guisa proposicional es, grosso modo, una proposición concebida como de una cierta forma lógica. La teoría de las guisas proposicionales permite una explicación unificada de la paradoja del análisis de Moore, la discriminación de la atención, y el incremento del conocimiento y la creencia a través de la exégesis de las proposiciones. ${ }^{25}$ Menciono esto aquí por el adicional poder unificador que tiene al subsumir bajo la misma explicación la diversidad de preguntas y la variedad de contextos epistémicos.

Siguiendo el enfoque de Platón y Powers, tomamos los diferentes poderes de una persona para contestar ciertas preguntas como determinando especies diferentes de conocimiento. Quizás algunas propiedades - ya sean cualidades o relaciones- de los objetos son últimas y verdaderamente inanalizables. Quizás algunas propiedades pueden analizarse totalmente en términos de propiedades primitivas. Sin embargo, la mayoria de las propiedades que consideramos en la vida diaria no parecen ser cabalmente analizables -en un número finitó de pasos-- en términos de propiedades primitivas últimas. Entonces, parece no haber ningún análisis último de muchas proposiciones que sabemos verdaderas. Por tanto, la jerarquía de las formas (y guisas) lógicas de la mayoría de las proposiciones no está compuesta (definitivamente) de un número finito de niveles. Así, la jerarquía de preguntas determinada por la mayoría de las proposiciones no es un sistema finito. Por tanto, hay en principio un número indefinido, e incluso infinitamente grande, de especies de conocimiento.

24 Véase en [11] cap. 3, la discusión de la jerarquáa de Jas formas lógicas de una proposición y de algunas de las leyes básicas que gobiernan dichas jerarquías. Véase el apéndice.

25 Para una discusión detallada de las guisas proposicionales y su aplicación al análisis de los juicios perceptuales, de los campos sensóreos, etc., véase [12], parte II. 
El resultado anterior parece explicar por qué carecemos en el lenguaje ordinario de un mecanismo sistemático para denotar o señalar las especies de conocimiento que nos conciernen. Obviamente, en la vida práctica no nos interesa la jerarquía total de las formas (y guisas) lógicas de una proposición, al menos no como tal. Las locuciones interrogativas que tenemos marcan puntos de interrogación. Al ponerlas en una oración interrogativa revelamos el nivel de la forma (y guisa) lógica y, dentro de esta forma, el punto de interrogación en el que estamos interesados. Compárense entre sí, por ejemplo, en el caso anterior, las preguntas: " $¿ E$ el caso que (2)?", "Si todos los vuelos fueran puntuales hoy, ¿dónde está Juan?", y "Si todos los vuelos fueran puntuales hoy, entonces, dado que Juan no está en la sala de espera ni en una caseta telefónica, ¿dónde está?"

Ahora surge la pregunta: si en el lenguaje ordinario carecemos de un mecanismo sistemático para especificar en qué preguntas y, a fortiori, en qué especies de conocimiento estamos interesados, ¿cómo nos las arreglamos? Pues al parecer nos las ingeniamos para comunicarnos acerca de lo que ciertas personas saben o no saben. La respuesta parece ser esta: logramos comunicarnos acerca de las especies de conocimiento exactamente de la misma manera en la que logramos comunicarnos acerca de las especies de otras propiedades, cuando necesitamos una especificación de un género (genus) con un número indefinido y quizás infinito de especies, a saber, el contexto de la comunicación determina las especies relevantes.

Lo que sostengo para 'saber' y 'conocimiento' es, en detalle, un poco diferente de lo que pasa con las palabras demostrativas como 'aqui' y 'alli' y de lo que pasa con las palabras para colores. Naturalmente, hay razones fundamentales para las diferencias. La tesis común es que, en sus usos primarios, todos estos tipos de expresiones son, en el fondo, indéxicas (véase [6]).

En el caso de 'aqui' y 'alli', las expresiones mismas y las oraciones en las que figuran necesitan un complemento por medio de una asociación con un lugar de proferencia posible (no real). En el caso de las palabras para colores, éstas y las oraciones que las contienen necesitan, en los usos perceptuales, una asociación con los tonos de color reales; pero pueden usarse de una manera genérica. Supongo que una razón de esto es que el color determinado real, que es el contenido de la experiencia, no suele ser importante en la comunicación. Así, en algunos usos, especialmente en los usos no perceptuales, las palabras para color no denotan un tono especifico determinado por el contexto, incluido el apuntar. Entonces, deben entenderse como denotando una cualidad genérica, determinable, o como implícitamente cuantificadas. Por ejemplo, 'el libro que está sobre la mesa en la oficina del director es rojo' puede tomarse como de la forma 'hay un tono $\phi$ idad, de la familia rojo, tal que el libro sobre la mesa del director es $\phi$ '.

Las palabras epistémicas 'saber' y 'conocimiento' pueden quizás usarse en 
un sentido genérico, tal y como se usan las palabras para colores. Como en el caso de estas palabras, el uso genérico descansa sobre el uso en el cual denotan especies determinadas, contextualmente precisadas. En el caso de 'saber' y 'conocimiento' no es el contexto perceptual, sino el contexto de investigación asumido el que precisa las especies determinadas relevantes con respecto a episodios de aseveración o de pensamiento.

En cada contexto de comunicación nos conciernen ciertas preguntas. Comúnmente no hay necesidad de formularlas, porque los cursos de acción con los que estamos comprometidos, o con los que planeamos comprometernos, exigen ciertas respuestas. Asi, el conjunto implícito de preguntas relevantes determina, por sí mismo, las especies de conocimiento que nos interesan. El contexto de comunicación y el contexto de investigación, ya sea que la inves. tigación la lleve a cabo una persona $u$ otra, determina, por tanto, un operador de especies, como los suscritos ' $Q$ ' y ' $R$ ' en la sección 15, que mapea el 'conocimiento genérico en especies relevantes de conocimiento.

Aparte de la investigación, una persona tiene, en un tiempo dado, los poderes epistémicos para contestar ciertas preguntas relativas a cierta proposición $p$. Entonces, independientemente de cualquier investigación, o de cualquier contexto de comunicación, una persona tiene conocimiento $\alpha$ de que $p$ para varias clases $\alpha$ de preguntas que incluyen preguntas relativas a esa $p$. El señalamiento lingüístico que hacemos es que en un contexto de habla dado, aun si no es comunicacional, un hablante, al escoger su tema de discurso, seleccionará al menos una clase $\alpha$ de preguntas, con respecto a la cual él atribuye conocimiento a las personas. Visiblemente, la selección de $\alpha$ es aquí, ante todo, lo que prepara, si no lo que dispara, un haz de disposiciones a pensar (y formular) ciertas preguntas. Entonces, la selección de una clase $\alpha$ de preguntas no necesita exhibirse en una lista con los miembros de $\alpha$, 0 en la formulación de una descripción de $\alpha$.

17. Otra dimensión de la semántica de 'saber' y de la teoria de las pret guntas: restricciones metodológicas y contextuales sobre los rangos de interrogación. Sostengo que cada proposición, o estado de cosas, determina una jerarquía de preguntas. Esta jerarquía está determinada simplemente por la jerarquía de las formas lógicas de la proposición (o estado de cosas) dada. Pero la jerarquía lógica total de las preguntas es, frecuentemente, demasiado amplia para los fines prácticos. Normalmente nos interesa un segmento modesto de la jerarquía interrogativa perteneciente a una proposición. El segmento en cuestión se separa de la jerarquía total por medio de un conjunto de restricciones. En un contexto de investigación dado, nos interesan preguntas que cumplen con ciertas condiciones, las cuales incorporan las restricciones relevantes. Tales condiciones son parte de las circunstancias que determinan la verdad de la proposición a la cual pertenecen la pregunta-cum-restricciones, así como la jerarquía de preguntas que la contiene. Obviamente, una per- 
sona puede tener el poder de encontrar una proposición $\mathrm{P}$ al pensar una pregunta $Q^{c}(\mathrm{P})$ con la restricción $c$ perteneciente a $\mathrm{P}$, y esa persona puede, aun así, carecer del poder para pensar $P$ al pensar una pregunta $R^{d}(P)$ con la restricción $d$ perteneciente también a $P$.

Las restricciones sobre las preguntas relevantes para una cierta investigación son parte del contexto de la investigación. En la medida en que una persona pueda estar simultáneamente involucrada en investigaciones diferentes, esa persona puede involucrarse en diferentes conjuntos de circunstancias normales para la verdad de las proposiciones de las investigaciones en cuestión. Más generalmente, el poder que una persona tiene para ofrecer una proposición $\mathrm{P}$ como la respuesta verdadera a una pregunta $\mathrm{Q}^{c}(\mathrm{P})$ puede no estar aparejado a un poder para dar $\mathrm{P}$ como la respuesta verdadera a una pregunta diferente $R^{d}(P)$. No obstante, el conjunto de circunstancias involucradas en la verdad de $P$, via la restricción $c$ y la pregunta $Q$, puede ser tan normal como el conjunto de circunstancias involucradas en la verdad de $\mathrm{P}$ vía la pregunta $\mathrm{R}$ y la restricción $d$.

En el ejemplo de Powers de las preguntas (Q) y (R) en la sección 15, no tenemos ninguna restricción metodológica sobre las preguntas; los dos contextos normales incluyen simplemente la capacidad de pensar las preguntas y la de usar las palabras en inglés. En este sentido los ejemplos son demasiado simples.

Echemos una mirada más detenida a las preguntas con restricciones.

Cada punto de interrogación en una guisa proposicional (es decir una proposición considerada como de una cierta forma lógica) determina, tanto un lugar en blanco en la proposición, por así decirlo, como un rango de posibles ocupantes de ese lugar. Por ejemplo, la proposición (2) de la anterior sección 16, analizada hasta donde la oración (2) permite, tiene el punto de articulación representado por la expresión 'vuelos', que produce, entre otras, las dos siguientes preguntas:

Q1) ¿Cuáles sucesos son tales que si todos ellos fueran puntuales hoy, Juan está en la sala de espera o en una caseta telefónica, o bien, no vino?

Q2) ¿Cuáles sucesos programados para tener lugar aquí son tales que, si todos ellos fueran (ocurrieran) puntuales hoy, Juan está en la sala de espera o en una caseta telefónica, o no vino?

Manifiestamente, un análisis del concepto vuelo indicará más puntos de interrogación dentro de este concepto en la proposición $\mathbf{P}$ expresada por la oración (2). Tales puntos de interrogación producirản preguntas ulteriores pertenecientes a $P$, con sus rangos propios de ocupantes.

La pregunta (Q1) determina un amplio número de ocupantes para el lu- 
gar en blanco representado por el pronombre en cursivas 'ellos'. Empero, el contexto de investigación en el que surge la pregunta puede dejar en claro que ciertos ocupantes no son relevantes. La pregunta $\left(\mathrm{Q}^{2}\right)$ reduce el conjunto de ocupantes al introducir algunas restricciones importantes. Sin embargo, esto puede ser todavía demasiado amplio para formular el problema del contexto real de investigación. Restricciones posteriores pueden hacer del conjunto de ocupantes relevantes un conjunto todavía más pequeño del rango total de los ocupantes lógicamente viables.

También puede haber exigencias metodológicas para las respuestas relevantes. Son exigencias externas que no necesitan tener nada que ver con la estructura lógica de una proposición, ni con la de cada una de las preguntas que la proposición determina en la jerarquía de preguntas. El contexto de interrogación puede establecer que una pregunta no está contestada por el simple hecho de encontrar la proposición a la que la pregunta pertenece. Puede requerirse que la proposición se encuentre de acuerdo con ciertos procesos - técnicas. Con el fin de $\operatorname{saber}_{\beta}$ que $p$, para algún conjunto $\beta$ de preguntas con restricciones metodológicas, uno debe tener no sólo el poder de pensar esa $p$ como la respuesta a meras preguntas en el conjunto $\beta$ : uno debe tener el poder de pensar esa $p$ como ajustándose también a las restricciones que conllevan las preguntas en el conjunto $\beta$.

Lo anterior resulta evidente por medio de una simple reflexión sobre: a) lo que cuenta como saber que Colón descubrió América el 12 de octubre de 1492, en un programa de adivinanzas en la televisión, comparado con b) lo que cuenta como saber eso en un ensayo de un estudiante de secundaria sobre el descubrimiento de América por parte de Colón, y comparado con c) lo que cuenta como saberlo en el caso de un historiador que defiende la fecha tradicional del descubrimiento frente a la afirmación ocurrente de algún célebre historiador de Harvard que sostiene que Colón descubrió América el 11 de octubre de 1492.

18. La jerarquia de los poderes epistémicos y los complejos fundamentales pregunta-proposición. El enfoque de Platón y Powers, tal y como se discutió y refrendó anteriormente, hace del conocimiento, no sólo un asunto acerca de creer una verdad con un cierto tipo de justificación, sino un asunto sobre la posesión del poder de encontrar una respuesta (verdadera) a ciertas preguntas. Esto exige una clarificación en términos del concepto de poder o habilidad usado aquí; las condiciones que activan estos poderes o capacidades; la relación de contestación entre la pregunta y la respuesta; lo que es para una persona creer que una cierta proposición es una respuesta (verdadera) a una pregunta, etc.

Ya hemos dado la teoría básica de las preguntas al desarrollar la concepción de una jerarquía de preguntas restringidas, determinada primero por las formas lógicas de una proposición, y luego reducida, tanto por la esti- 
pulación de condiciones sobre los ocupantes de los lugares en blanco en los puntos de interrogación, como por las restricciones metodológicas. Naturalmente, esta teoría de las preguntas necesita un desarrollo adicional. Pero lo que hemos presentado anteriormente es suficiente para las secciones elementales de la epistemología básica. Aquí optamos por la terminología simple: una respuesta $P$ a una pregunta $Q$ es una proposición verdadera $P$, tal que $P$ satisface las restricciones sobre $Q$ y $Q$ pertenece a $P$ en el sentido explicado arriba, a saber: $Q$ surge de $P$ por la presencia de un lugar en blanco en una guisa proposicional de $\mathrm{P}$, donde el lugar en blanco determina un subconjunto de todos los ocupantes lógicamente posibles para ese espacio en blanco. Entonces, 'respuesta verdadera' y 'respuesta' significan lo mismo.

Ahora considérese lo siguiente:

1) En el tiempo $t$ Juan creyó que 'deny' era (es) una palabra de cuatro letras (en inglés) terminada en E, ENE y YE.

2) En el tiempo $t$ Juan creyó que 'deny' era (es) una respuesta (incompleta) a la pregunta "¿Qué palabra (en inglés) de cuatro letras termina en E, ENE y YE?”

Estas oraciones pueden tomarse, literalmente, por lo que muestran, esto es, como si tuvieran internamente (en una terminología que no me gusta, muchos autores dirían de dicto) todas las expresiones de la cláusula subordinada. Si se toman así, entonces se sostiene que el Juan del que se habla en (1) y (2) cree las proposiciones formuladas en las cláusulas-qué subordinadas. En esa interpretación, (1) y (2) expresarian proposiciones verdaderas si Juan puede entender la pregunta y pensar al mismo tiempo en 'deny'. Entonces, en la interpretación sintáctica interna, ni la oración (1) ni la oración (2) describen la aptitud de Juan para dar 'deny' como un ejemplo de una palabra que termina en E, ENE y YE, cuando se le pide hacerlo.

Quizá podemos expresar que Juan tiene esa aptitud tomando la expresión 'deny' en (1) y (2) como si figurara externamente (o bien, de nuevo en una terminología que no me gusta, de re.) ${ }^{26}$ En esta interpretación, (1) y (2) no son perspicuas, pues representan lo que se expresa mejor de la siguiente manera:

1a) En el tiempo $t$ Juan creyó de 'deny' que era una palabra de cuatro letras (en inglés) que terminaba en E, ENE y YE.

2a) En el tiempo $t$ Juan creyó de 'deny' que era una respuesta (incomple-

26 Véanse en [7] algunas de las razones para preferir la terminología 'interna' -'externa' a la terminología 'de dicto'- 'de re' al referirse a las figuraciones de expresiones en construcciones en oratio obliqua, y para una discusión de la transparencia proposicional y de la opacidad proposicional. 
ta) a la pregunta "¿Qué palabra (en inglés) de cuatro letras termina en E, ENE y YE?”

Estas oraciones, en su uso perspicuo, asignan la referencia del verbo "deny" al usuario de la oración total (1a) o (2a). Las oraciones (1) y (2), por otra parte, representan en sus interpretaciones internas una acumulación de referencias, hechas tanto por parte del usuario como por la del Juan de quien se habla. Así, (1a) y (2a) dejan sin especificar cómo se refiere Juan al verbo 'deny': dejan sin especificar cuál es, exactamente, la proposición que se dice que Juan cree; y de esta manera, son proposicionalmente opacas con respecto al sujeto de la proposición creída por Juan. Por tanto, las oraciones (la) y (2a) pueden expresar verdades cuando (1) y (2), interpretadas internamente, expresan verdades; pero pueden también expresar verdades cuando (1) y (2) no expresan verdades, debido a que Juan se refiere al verbo 'deny' de otra manera que cuando usa la expresión '(el verbo) 'deny'.

Sin embargo, ni (1a) ni (2a) describen la aptitud de Juan para dar 'deny' como un ejemplo de una palabra que termina en E, ENE y YE cuando se le pide hacerlo. ${ }^{27}$ Esta aptitud no es idéntica a -ni parte de_ la creencia, por parte de Juan, de que 'deny' está compuesta de DE, E, ENE y YE y su aptitud para entender la pregunta "¿Qué palabra (en inglés) de cuatro letras termina en E, ENE y YE?"

Sin importar en qué aptitudes pueda consistir un estado de creencia de que $p$, la anterior discusión del ejemplo de Powers, y su generalización a todos los tipos de preguntas con restricciones, dejan en claro que la aptitud para dar una respuesta a una pregunta no consiste en, pero sí presupone, la comprensión de la pregunta y la creencia en la proposición que contesta la pregunta. La aptitud de contestar una pregunta tiene que ver con la aptitud de uno para reunir pruebas en favor, y esto, a su vez, tiene que ver con la organización de las propias creencias en las profundidades inconscientes de la mente. El conocimiento tiene que ver con las estructuras de las pruebas en favor. Claramente, como ya hemos insinuado, una explicación pasiva del conocimiento, sólo en términos de creencias y verdades, no puede ser adecuada; el análisis del conocimiento debe conectar las creencias de una persona con sus aptitudes para reunir pruebas en favor.

El principio de Platón y Powers de que el conocimiento es poder, en par-

27 Parece que Powers tiene una opinión diferente de las oraciones (1) y (2), si podemos interpretar que acepta para 'cree' lo que sostiene para 'sabe', a saber: "De nuevo, usamos ' $x$ sabe que la respuesta a la pregunta $Q$ es $P$ ' no para decir que, si se le preguntara ' $E$ E $P$ la respuesta a $Q P^{\prime}, x$ contestaría sí; sino, por el contrario, para decir que, si se le preguntara $Q, x$ contestaria $P$. Así, lo que llamamos 'saber que la respuesta a $Q$ es $P$ ' es algo más que solamente saber (aceptar correctamente) la proposición de que la respuesta a $Q$ es P." ([50], 348; cursivas del autor.) 
ticular el poder de contestar preguntas, es, por tanto, un criterio importante de la adecuación de cualquier análisis del conocimiento.

Sócrates creyó que el conocimiento es poder en un sentido mucho más fuerte que el entronizado en el principio de Platón y Powers sobre el conocimiento. Para Sócrates, el conocimiento es un poder de actuar, de producir movimientos físicos diferentes de las actividades cerebral y vocal requeridas, causalmente, para pensar y afirmar. Sócrates pudo haber estado en lo correcto, y una teoría comprehensiva del conocimiento debería incluir la subteoría acerca de las conexiones generales entre el conocimiento y la acción. Estas conexiones generales incluyen no sólo las más fundamentales, por ejemplo, que toda proposición que una persona cree, a fortiori, toda proposición que una persona sabe, es una premisa posible, tanto para la adquisición de creencias posteriores como para la determinación de lo que él o ella debe hacer. Las conexiones generales entre el conocimiento y la acción incluyen relaciones causales entre las creencias y los poderes que componen, por una parte, el conocimiento, y por la otra, la producción de la acción. Estas conexiones causales incluyen, pero no agotan, el fenómeno de la volición que funde la acción de una persona con su conocimiento de qué hacer. ${ }^{28}$

La epistemología básica, de la que aquí nos ocupamos, debe tratar la muy fundamental y difundida conexión entre el conocimiento y la acción. Ésta es la conexión encajada en nuestros poderes para pensar respuestas a las preguntas. Estos son los poderes epistémicos por excelencia. Dependen de la organización de nuestras creencias; su ejercicio, a la vez, reorganiza nuestras creencias. Pero esta organización y reorganización de creencias que se alimenta y resulta, respectivamente, de nuestro pensar preguntas y de buscar sus respuestas, no es, en sí misma, una creencia, ni aun la creencia adicional de que las demás creencias están en un cierto ordenamiento jerárquico. La organización comprobatoria de las creencias, producida por el hecho de enredarse en preguntas y respuestas, es una organización causal de los grados en los que uno está dispuesto a pensar (afirmativamente, en su mayor parte) esta o aquella proposición.

En suma, nuestros poderes epistémicos más básicos tienen que ver con el ordenamiento causal de los grados en los que estamos dispuestos a producir premisas que podemos necesitar en nuestros razonamientos. Debido a que nuestros razonamientos pueden llevar a cursos de acción diversos, nuestros poderes epistémicos más básicos están en los cimientos de la conexión entre el conocimiento (y creencia) y la acción.

Los poderes epistémicos básicos consisten en poderes para pensar preguntas, para pensar las respuestas correspondientes y para que el mismo acto de pensar las preguntas lleve a pensar las respuestas. Uno no necesita tener el

28 Para una explicación de la volición véase [11], cap. 10, y el capítulo 12 para una discusión de los diversos tipos de acción intencional. 
poder lingüistico adicional para usar las palabras de segundo orden "respuesta" y "pregunta". Considérese, por ejemplo, el caso de Amy, una niña muy pequeña. Un visitante le pregunta: "¿Dónde está tu muñeca, Amy, la que te trajo Santaclós?" Y Amy, incapaz de articular la respuesta, toma al visitante de la mano y la lleva a su cuarto en el piso de arriba y dice: "Aquí, muñeca." ${ }^{2}$ Sin duda alguna, Amy puede pensar proposiciones, puede pensar preguntas y puede pensar proposiciones como respuestas a preguntas. Estas capacidades de pensamiento tienen, naturalmente, un fundamento en sus poderes lingüísticos. Pero las capacidades de pensamiento, no obstante que puedan estar fundadas en el poder y el control lingüísticos, llevan ventaja al desarrollo lingüístico.

Ahora bien, junto a los poderes de articulación debemos distinguir entre el pensamiento de primer y el de segundo orden. La aptitud de pensamiento fundamental que uno necesita es simplemente la aptitud de pensar proposiciones y preguntas de primer orden. Así, uno debe ser capaz de pensar, digamos, a) Juan está feliz, b) ¿Está feliz Juan? y c) ¿Quién está feliz?, para "ver" que (a), si es verdadera, está en una relación especial con (b) y (c). Dicho de otra manera, uno puede pensar la (A) y la (B) siguientes:

A) ¿Está Juan feliz?... [Examen de creencias y percepciones.] Sí, Juan está feliz.

B) ¿Quién está feliz?... [Examen de creencias y percepciones.] JAh! Juan está feliz.

Uno puede hacer esto $\sin$ ser capaz de pensar ninguna de las siguientes cosas:

$A+)$ Pregunta: “Está feliz Juan?”... [Examen de creencias y percepciones.] Respuesta: "Juan está feliz."

$B+$ ) Pregunta: “¿Quién está feliz?”... [Examen de creencias y percepciones.] Respuesta: "Juan está feliz."

Lo que necesitamos para la fundamentación de nuestros poderes epistémicos es únicamente el poder para hacer una conexión operacional entre las respuestas a preguntas, como en (A) y (B). Estas ilustran la dialéctica primaria del pensamiento. Supongo que podemos hablar de estas conexiones operacionales, como la conexión $; A h$ ! - Si o, simplemente, la conexión Sf entre una pregunta y una proposición tomada como una de las respuestas a la pregunta. Esta conexión operacional debe distinguirse cuidadosamente de la relación predicativa que uno puede contemplar pasivamente como establecida entre una pregunta y cada una de sus respuestas. Esta relación es la

29 Debo este ejemplo a Miriam M. Castañeda. Véase también [8] para una doble generalidad del lenguaje. 
pensada en $(\mathrm{A}+)$ y $(\mathrm{B}+)$. Esta relación está expresada por la locución predicativa es una respuesta a y, naturalmente, las oraciones que expresan las proposiciones de segundo orden donde se contiene esta relación requieren nombres para flanquear 'es una respuesta a'. Considérese, por ejemplo:

3) "Juan está feliz" es una respuesta a "¿Quién está feliz?"

Aquí las comillas forman nombres. Por otra parte, la expresión "Sf", que, según acabamos de estipular, es un signo de la conexión operacional subyacente a (3), no necesita nombres, sino que se aplica directamente a las oraciones y quizá forma con ellas una oración que expresa una proposición compuesta. Así, no necesitamos comillas ni ningún otro expediente nominalista y podemos escribir simplemente:

3*) ¿Quién está feliz? Sf Juan está feliz. para representar la conexión operacional subyacente a la relación de respuesta denotada en (3).

En general, propongo usar la palabra 'Sf' como un símbolo técnico para el conectivo más básico entre una pregunta y una proposición que puede pensarse como una respuesta a esa pregunta. Así, tenemos complejos preguntaproposición de la forma:

4) ¿Q? St $p$.

Una vez más, los complejos de la forma (4) no son enunciados relacionales de segundo orden como (3): son complejos de primer orden. No son preguntas. Pueden verse como proposiciones, pues al parecer los creyó una persona que razona como en las (A) y (B) anteriores. Los principios primordiales que rigen los valores de verdad para tales complejos son éstos:

$\left(Q p . \mathrm{T}_{0}\right)$ Un complejo pregunta-proposición de la forma " $q S f$ " es verdadero sólo en caso de que la pregunta $q$ pertenezca a la jerarquía de preguntas determinadas por la proposición $p$; de otra manera es falso.

$\left(Q p . \mathrm{T}_{i}\right.$ ) Un complejo pregunta-proposición de la forma " $q S f_{i} p$ ", donde $i$ denota, o señala, un conjunto $\alpha_{i}$ de restricciones, es verdadero sólo en caso de que la pregunta $q$ pertenezca a la subjerarquía de preguntas determinada por $p$ y esté gobernada por las restricciones en $\alpha_{i}$; de otra manera, el complejo " $q S f_{i} p$ " es falso.

No tenemos espacio aquí para desarrollar la lógica fundamental de los complejos pregunta-proposición. Obviamente, ningún análisis del conocimiento dentro de los lineamientos platónico-powersianos, seguidos aquí, puede ser completamente esclarecedor hasta que esa lógica sea, también, completamente desarrollada.

Evidentemente, un ente pensante puede ser capaz de pensar complejos pregunta-proposición, aun si no es capaz de pensar las correspondientes pro- 
posiciones relacionales clasificatorias de segundo orden acerca de las preguntas y sus respuestas. Por ejemplo, un niño pequeño puede ser capaz de pensar complejos como (3*), pero no proposiciones como (3). Así, hay una jerarquía de poderes epistémicos básicos: en el fondo, el poder epistémico crudo es el poder de ser llevado a pensar que $p$ por el acto mismo de pensar la pregunta $q$. Este es el poder que los niños, y quizá los primates y animales domésticos sofisticados, adquieren primero. Después está el poder para pensar complejos de la forma " $q S f$ " y " $q S f_{i} p$ " con restricciones que solamente exigen la normalidad de las circunstancias asumidas. Después aparece el poder de pensar complejos de la forma " $q S f f^{\prime}$ " y " $q S f_{i} p$ " con restricciones anormales y, por último, los poderes para pensar las restricciones más sofisticadas. En algún punto del desarrollo entre la normalidad y la anormalidad surge el poder de alcanzar un metalenguaje: tener pensamientos de tipo relacional con la forma "la pregunta $q$ tiene una respuesta: (la proposición) $p "$.

Esta jerarquía de poderes epistémicos deja lugar a los usos amplios y estrechos del vocabulario epistémico, especialmente de las palabras 'saber' y 'conocimiento'. ¿Saben los gatos o perros domesticados, por ejemplo, que su dueño está en casa, está enojado o está cariñoso? Todo esto depende de qué capacidades de pensamiento atribuyamos a los gatos y cuáles a los perros. ¿Y a los niños pequeños?

19. Las circunstancias de verdad de la verdad conocida, implicadas en las restricciones a las preguntas, no necesitan conectar causalmente al conocedor con esa verdad. Suele darse por sentado que para que una persona sepa que $p$ no necesita haber una causa común de que la persona crea que $p$ y de $p$, ni una via de causación de $p$ a que la persona crea que $p .^{30}$ Creo que esto es correcto.

No podemos revisar los argumentos en favor o en contra de la explicación causal del conocimiento. Para el lector atento resultará obvio que algunos de los ejemplos dados anteriormente, especialmente las subseries del tramposo no normal, presentan problemas a la mayoría de las explicaciones causales propuestas. Sin embargo, nuestro interés reside aquí en poner de manifiesto algunas características generales de los conexos epistémicos de justificación.

El ciego Tiresias. Tiresias, un ciego adivino, tiene el extraordinario poder de ver visiones muy vívidas en su imaginación. Esto sucede después

30 Véanse, por ejemplo, Skyrms [58], Sosa [61], Paxson [49], Pappas [44], Lehrer [40]. Una de las primeras explicaciones causales del conocimiento es la propuesta por Goldman en [27]. Skyrms la atacó con un ejemplo que los autores posteriores consideraron concluyente. Loeb, en [42], lleva a cabo una importante aclaración del argumento de Skyrms y muestra que no es tan devastador como se pensó. La serie del tercer tramposo no normal muestra la insuficiencia del principio causal de Goldman. 
de que ha comido pepinos escabechados con una mezcla de tequila y algunos preparados muy secretos. Tiresias dice que sus visiones traen una fecha en la parte inferior. Se ha encontrado que sus visiones corresponden, punto por punto, a sucesos del pasado, a sucesos del futuro o a sucesos simultáneos a sus visiones, según las fechas que ve. Sin embargo, no hay manera alguna en la que esos sucesos puedan conectarse causalmente con las visiones de Tiresias. Este poder le vino a Tiresias, según recuerda, el día que cumplió 25 años. Durante unos tres años, no prestó atención a sus visiones. Pero después algunas noticias de los periódicos describieron lo que habia visto, y entonces comenzó a creer que sus visiones fechadas correspondían a la realidad.

¿Sabe Tiresias que su visión actual es verdadera? ¿Lo sabemos nosotros, que lo hemos examinado cientos de veces sin descubrir un solo error? Necesitamos recurrir de nuevo a la noción de circunstancias normales, estables. Las primeras creencias de Tiresias no estaban fundadas de ninguna manera. Sus visiones posteriores están fundadas sobre la generalización de que, ceteris paribus, sus visiones representan la realidad, esto es, dado el supuesto de un contexto normal de circunstancias que conecta la ocurrencia de sus visiones con lo que éstas describen. Si la generalización relativa a las circunstancias normales, descritas sólo en parte en El ciego Tiresias, vale, entonces su creencia corresponde a la realidad y, me parece, constituye conocimiento.

Quizá las circunstancias normales de Tiresias incluyen elementos de los cuales no tenemos ninguna idea. De ser así, y sin importar cuán extravagantes puedan ser estos elementos, el supuesto implícito de que las circunstancias son normales permanece válido. Si algunas circunstancias extravagantes afectan la normalidad del contexto, entonces Tiresias no sabrá, aun si estas circunstancias vienen en pares equilibrados, justo como sucede -acaso el lector lo recuerde- en Las series del tramposo. Entonces, si Tiresias tiene conocimiento de (o tiene una creencia razonable acerca de) esas circunstancias y las considera en sus pretensiones sobre la verdad de sus visiones, Tiresias sabe de nuevo que su generalización revisada, es, por hipótesis, verdadera.

$N o$ es necesario que Tiresias sepa que sus creencias están causalmente conectadas con lo que cree. Esto es más claramente así, cuanto más detalladas sean sus visiones y creencias acerca de los sucesos representados en sus visiones. En esta coyuntura aparecen problemas serios y profundos acerca de cómo puede él pensar sobre sucesos con los que no puede estar causalmente conectado. Indudablemente, si los contenidos de la conciencia son en esencia universales, entonces Tiresias, como cualquier otro, piensa en todos los tipos de particulares por medio de rasgos o caracteristicas, y de sus relaciones consigo mismo y con los particulares presentes en sus campos perceptuales. ${ }^{31}$ Estos

31 Véanse [8] y [12] para explicaciones de la naturaleza de nuestra conciencia de los particulares. 
asuntos son difíciles, pero una teoria epistemológica comprehensiva debe ocuparse de ellos.

20. Circunstancias normales de verdad y el orden del mundo. Hemos regresado al supuesto más crucial y más intimo que subyace a nuestras pretensiones cognoscitivas, a saber: que las condiciones de verdad de las proposiciones sabidas son normales o tienen anormalidades identificables. Ya hemos formulado en las secciones 13-17 algunos de los principios más fundamentales que rigen el papel epistémico de la normalidad. Digamos algo acerca de lo que es la normalidad. Para comenzar, el principio más general y fundamental es:

PCN* La presuposición clave acerca de la normalidad.

Los cambios en nuestro entorno inmediato tienen un orden confiable determinado por: 1) una jerarquía de principios y leyes generales que no podemos especificar en su mayor parte (al menos por el momento) y 2) un conjunto de regularidades especificas relevantes, que podemos especificar, y tanto el orden general como las regularidades específicas permanecen funcionando al menos durante el periodo que nos concierne.

La idea de que hay en el mundo un orden subyacente que perdura, pero que no podemos especificar en un momento dado, es el supuesto permanente que no sólo unifica cada vida personal, sino que también nos unifica a todos como miembros de una comunidad epistémica. El supuesto de un intimo orden universal que sustenta nuestras acciones, pero que no podemos especificar más allá de algunas regularidades específicamente relevantes, es necesario para planear nuestras acciones y para contar con nuestra capacidad de llevar a cabo nuestros planes. Dada nuestra ignorancia completa de los cambios del mundo necesitamos el supuesto clave PCN*. Es un supuesto muy económico: podemos llevar a cabo nuestras tareas sin detenernos a formular los principios que rigen el orden del mundo. Es un supuesto vital, ya que debemos vivir y hacer nuestras cosas, independientemente de cuánto conocimiento del orden del mundo tengamos, e independientemente de cuánto tiempo tengamos para encontrar cuál sea la estructura de ese orden.

No podemos saber que el mundo está totalmente ordenado. Algunos de nosotros, los científicos y filósofos, descubrimos cada vez más principios del orden del mundo. Pero carecemos del poder para contestar todas las preguntas acerca del orden del mundo, especialmente si ponemos algunas restricciones metodológicas arduas a nuestras preguntas, como las que ahora se aplican en las ciencias naturales. Así, el principio fundamental PCN* es parte del marco no especificable dentro del cual determinamos que una cierta persona sabe ciertas verdades. Por otra parte, el supuesto fundamental y los otros supuestos estructurales que constituyen los marcos dentro de los cuales las creencias resultan justificadas, o no, no pueden justificarse ellos mismos dentro de esos marcos. Si hay marcos más abstractos, o más comprehensivos, dentro 
de los cuales podemos preguntar si el PCN* está justificado o no, es un tema muy importante, pero que está más allá de la epistomolgía básica. En todo caso, tenemos el principio:

NSSJ. Los supuestos o presuposiciones estructurales acerca del mundo, o de una parte suya, que determinan los marcos epistémicos, no son autojustificables ni son justificables en los marcos que determinan.

Este principio enlaza el carácter gestáltico del conocimiento, introducido en la sección 2, y la determinación contextual de las especies de conocimiento, discutida en la sección 15. Para que una persona sepa algo debe tener algún arsenal de creencias, pero estas creencias, que por converger sobre otras creencias les confieren el status de conocimiento $\alpha$, no son ellas misma conocidas $\alpha$.

Varios supuestos penetrantes rigen el papel epistémico de las circunstancias de verdad. Son de diferentes tipos: algunos estipulan características generales del orden del mundo, otros estipulan conexiones entre el orden del mundo y la mente. Los siguientes son sólo algunos de los más discutidos:

PE. El principio de la experiencia. Toda proposición que una persona está justificada en creer debe ser asequible para la persona a través de la experiencia.

PBE. El principio de la base empirica. La más fuerte asequibilidad a través de la experiencia de una proposición $P$ para una persona $S$ en un tiempo $t$ es que $P$ se halle totalmente presente en una experiencia presente y ostensible de $S$ en $t$, i. e., que $P$ sea en $t$ el contenido total de un episodio de conciencia de $S$, o el contenido total de un segmento de tal episodio.

PR. El principio racionalista. Las proposiciones asequibles a una persona $\mathbf{S}$ no necesitan ser los contenidos exhaustivos de los episodios de conciencia de S (como en PBE), en el tiempo $t o$ durante un conjunto de tiempos, pero entonces tales proposiciones deben estar conectadas con los contenidos experienciales de $S$ en algún tiempo, y con las creencias de $S$, por medio de una red de principios que componen, en parte, el orden del mundo.

PLK. El principio de Leibniz y Kant. Tanto los principios de la lógica deductiva como los principios que rigen las relaciones estructurales entre conceptos (o propiedades) son principios del orden del mundo.

POJM. Principio del orden jerárquico del mundo. Las diferentes categorías de entidades o estados de cosas que componen el mundo están conectadas, unas con otras, por los principios de la clasificación de las categorías, el ascenso de una categorfa a 
otra superior, el descenso de una categoría a otra inferior y la conmutación a través de categorías no ordenadas linealmente. Estos tipos diferentes de principios conectan: a) las experiencias sensibles con los objetos físicos; $b$ ) los estados de cosas físicos con los estados de cosas mentales; c) los objetos percibidos con los no percibidos; d) los estados de cosas experienciables con los teóricos; $e$ ) las proposiciones particulares con las generalizaciones; $f$ ) los testimonios presenciales con los asuntos examinados; $g$ ) los hechos con los valores; $h$ ) los valores con las obligaciones y derechos; etc.

PNIAOM. El principio de la naturaleza inferencial de nuestra actitud hacia el orden del mundo. Tendemos (por economía del pensamiento y de la acción) a tratar los principios del orden del mundo que reconocemos como si estuvieran montados sobre mecanismos de inferencia, más que como creencias formulables y premisas explícitas de razonamientos. Los principios del orden del mundo más abstractos y penetrantes (como los enlistados aquí) son rara vez, si es que alguna, objeto de reflexión, aunque estructuran la mayor parte de nuestro razonamiento y pensamiento. ${ }^{32}$

PHH. El principio de Heidegger y Hahn. Siempre nos encontramos enmedio de un mundo que tiene un orden general asumido, y tenemos una concepción de algunos aspectos de ese orden.

PDH. El principio de Duhem y Hahn. El orden del mundo no está fijado para todos los tiempos; puede cambiarse a voluntad, pero sólo por trozos.

PQ. El principio de Quine. La jerarquía del orden del mundo para una persona $S$ en un tiempo $t$ es una jerarquía de los grados de disponibilidad de $\mathrm{X}$ para resistirse a abandonar una proposición dada. ${ }^{33}$

AD. El axioma de Descartes. Las proposiciones tipo cogito acerca

32 Para una discusión fascinante de los diferentes papeles de los principios de inferencia, especialmente su papel en la estructuración de los conceptos, véase Sellars [53]. Una discusión penetrante de la inferencia aparece en Harman [29].

33 Puede parecer impropio formular el axioma de Quine en términos de proposiciones, en lugar de formularlo en términos de oraciones. Pero no estamos usando la palabra "proposición' para significar ni una verdad ni una falsedad, y obviamente las proposiciones en este sentido no son oraciones. De hecho, no son ni siquiera clases de oraciones equivalentes, bajo una equivalencia u otra, como se muestra en [11], nota 34. Sin embargo, todavía dejamos abierto el que las verdades (y falsedades) puedan reducirse a oraciones 0 , mejor, a clases de oraciones (con el fin de permitir equivalencias de intralenguaje y de interlenguaje), junto con algo más. Si el estudioso de Quine no está todavía a gusto con la palabra 'proposición', puede tomar el axioma anterior como un axioma semejante al de Quine, más que como uno del mismo Quine. 
de una persona $S$ y un tiempo $t$, para el efecto de que $S$ tiene en ese momento ella misma, en $t$, tales y cuales experiencias, son máximamente resistentes a ser abandonadas por $S$ en $t$. [Aquí 'ella misma' y 'en ese momento' son cuasi-indicadores.] ${ }^{34}$ PC. El principio del cogitatum. Las proposiciones tipo cogitatum pertenecientes a una persona $S$ y a un tiempo $t$ para el efecto de que dentro del campo de conciencia total de $S$, incluidos los campos perceptuales de $\mathrm{S}$, tal y cual cosa ocurre, son máximamente resistentes a su abandono por $\mathrm{S}$ en $t$.

Estos principios generales no nos dan las regularidades específicas que una persona pueda usar como premisas para formar su conocimiento del mundo. Ellos, junto con otras generalidades similares, solamente constituyen el esquema más amplio dentro del cual colocamos principios y leyes menos penetrantes, y más definidos y rigurosos. Todos estos principios y leyes constituyen, entonces, el marco dentro del cual las creencias de una persona han de cohesionar.

Las anormalidades de las circunstancias de verdad de una proposición dada que versa sobre asuntos de hecho particulares, tienen siempre que ver con regularidades específicas dentro del marco general. Proposiciones más generales pueden implicar anormalidades que giran en torno a regularidades más generales. Leyes más generales pueden estar implicadas en las anormalidades de las circunstancias de verdad en las que se consideran las leyes. Finalmente, los principios, aparentemente inocuos, enlistados anteriormente, y su familia, pueden producir anormalidades, al menos meramente pensables, en los contextos de verdad donde se examinan las leyes de la naturaleza más generales, $\mathrm{u}$ otros principios fundamentales. Este ordenamiento jerárquico de las anormalidades, reales o solamente pensables, de las circunstancias de verdad relevantes para la justificación de la creencia, es simplemente una consecuencia de la estructura jerárquica del orden del mundo.

Apenas hemos tocado la superficie del vasto tema deslas circunstancias de verdad normales relevantes. Pero esto debe bastar para nuestras presentes reflexiones sobre la epistemología básica. Debemos llevar estas reflexiones a un fin entrelazando los diversos hilos del tejido del conocimiento que hemos desplegado con vistas a dar una mirada preliminar a la urdimbre del conocimiento.

34 Para los papeles referenciales caracteristicos y cruciales de los cuasi-indicadores, véanse [6], [7] y [12], II. 9. Para una interpretación anticartesiana del cogito ergo sum, véase [12], II.4. 


\section{II \\ Un andisis preliminar del conocimiento de que $\mathrm{p}$}

1. Los elementos de los contextos epistémicos. Hemos visto en nuestra exégesis de los datos en la parte I cómo aparecen las especies de conocimiento de que $p$ y cómo esas especies están determinadas por diferentes parámetros. Hemos señalado cómo hay un marco de creencias que se da por sentado, el cual revisa la superficie de un contexto normal asumido, y cómo hay creencias explícitamente formulados o formulables que constituyen elementos específicos de prueba y justificación de otras creencias. Es el status de estas últimas creencias el que está en discusión. Hicimos notar cómo un conjunto de preguntas con restricciones, que comúnmente se asume y cuya revelación se deja al contexto de la comunicación, determina tanto un conjunto de fines epistémicos como un conjunto de poderes epistémicos posibles. También señalamos que nuestras creencias dependen de - $\mathrm{e}$ incluyen - las creencias ajenas, a pesar de la desconfiabilidad inherente a los informes de los demás.

En resumen, un contexto epistémico es un séxtuplo ordenado:

$$
C_{i}=\left\langle F_{i}, A_{i}, S_{i}, R_{i}, Q_{i}, P_{i}\right\rangle \text {, donde: }
$$

1. $F_{i}$ es el conjunto de "hechos" presupuestos: las creencias relevantes que acerca de los problemas pertenecientes a $P_{i}$ tienen los miembros de $A_{i}$, incluyendo las creencias acerca de lo que otros miembros de $A_{i}$ creen y dicen, si el testimonio de los demás es pertinente.

2. $A_{i}$ es el conjunto de agentes de la comunidad epistémica pertinente implicada en la decisión de los problemas pertenecientes a $P_{i}$.

3. $S_{i}$ es la red jerárquica de presuposiciones y supuestos estructurales, aceptados al menos por algunos miembros de $A_{i}$, que según se presume son pertinentes para la determinación de la verdad o falsedad de las proposiciones en $\boldsymbol{P}_{\boldsymbol{i}}$ por parte de los miembros de $\boldsymbol{F}_{\boldsymbol{i}}$ y $\boldsymbol{R}_{\boldsymbol{i}}$ : son las presuposiciones y supuestos que funcionan en la economía mental de las personas en $A_{i}$ como mecanismos de inferencia, más que como premisas principales.

4. $R_{i}$ es el conjunto de aspectos pertinentes de anormalidad aplicables a $S_{i}$.

5. $Q_{i}$ es el conjunto de preguntas, regido quizá por algunas restricciones sobre el rango de los lugares en blanco en las preguntas y por algunas restricciones metodológicas.

6. $P_{i}$ es el conjunto de proposiciones cuyo status epistémico está en discusión: ellas son las posibles respuestas a las preguntas en $Q_{i} \cdot{ }^{35}$

35 Como se explicó en la anterior nota 2, la inspiración principal de la explicación de los contextos epistémicos de justificación desarrollada aquí, es la explicación de los contextos de justificación de las acciones, intenciones y normas propuesta en [11], caps. 5, 6 
2. Un análisis tentativo del conocimiento de que $p$. La propuesta que sigue es sólo tentativa. No estoy ni siquiera seguro de que una definición explícita de ' $X$ sabe que $p$ ' sea la consumación que debemos esperar en la epistemología básica. Como he argumentado en otro lugar, el método definitorio de la filosofía analítica clásica, a pesar del poder iluminador que pueda alcanzar, no puede aportar la clarificación filosófica total que algunos deseamos. Esto es en parte así porque las definiciones están sujetas a la regla estricta de dar conjuntos de condiciones que sean necesarias y suficientes. Así, podemos fácilmente omitir la luz que el descubrimiento de condiciones que son necesarias pero no suficientes, o viceversa, puede arrojar. ${ }^{36}$

Naturalmente, las definiciones de ciertos conceptos son, algunas veces, factibles. Además, podemos ofrecer aquí un análisis tentativo del conocimiento de que $p$ que supera el análisis estándar en varias coyunturas. Primero, proporcionamos un desarrollo del principio platónico-powersiano de que el conocimiento cognoscitivo tiene que ver, no sólo con las creencias, sino también esencialmente con los poderes para contestar preguntas. Segundo, asumimos la teoría de las preguntas bosquejada en las secciones 16-18. Tercero, tomamos en consideración la multiplicidad de especies de conocimiento y su especificación indéxica por los contextos epistémicos. Cuarto, reunimos en nuestro análisis la característica crucial de la normalidad de un contexto y sus aspectos de anormalidad. Desde luego, no estamos dando, además, un análisis fichteano del conocimiento. Tampoco requerimos operaciones probabilísticas; pero la probabilidad puede incluirse en las restricciones a las preguntas en $Q_{i}$. Recuérdese, sin embargo, que el esquema de análisis es sólo la punta del iceberg descrito en la parte $I$.

Ahora, vayamos a fondo: recuérdese que $q \mathbf{S f}_{\mathbf{i}} p$ significa, como se explicó en la sección 18, la proposición no clasificatoria de primer orden de que $q$ (pregunta) tiene a $p$ (proposición) como una respuesta (verdadera); recuér-

y 8. Debo hacer notar un error en la explicación anterior, el cual me fue amablemente señalado por Michael Bratman. Este error consiste en no haber puesto en un contexto de acción el importante parámetro que consiste en un conjunto de acciones primeras, es decir, acciones que son para el contexto en cuestión como átomos de acción - aun cuando en otros contextos puedan analizarse, ya sea como acciones compuestas puras, cuyos componentes son nada más acciones, o como acciones mixtas que tienen algunos componentes proposicionales. Bratman probó el importante teorema de que un contexto que tiene, como acciones primeras, acciones que están compuestas de acciones primeras, puede llevar a contradicciones. El error epistemológico de la contraparte se evita en este ensayo haciendo que cada contexto epistémico $C_{i}$ de justificación de la creencia contenga el parámetro crucial $\boldsymbol{P}_{\boldsymbol{i}}$. En el caso epistémico, la explicación de las razones en las que se funda este parámetro es, afortunadamente, aun más obvia: $P_{i}$ es, como se notó, el conjunto de respuestas, ya sea verdadero o falso, a las preguntas en $Q_{i} . Y$ a la inversa, dada la teoria de las preguntas bosquejada aqui, los miembros de $P_{i}$ son las proposiciones que producen las jerarquías de preguntas que contienen las preguntas en $Q_{i}$. Ast, la pertinencia de $P_{i}$ a $Q_{i}$ es obvia y profunda.

36 Véanse [9] y [10] como discusiones complementarias del papel de las definiciones en filosofía y para una crítica del método clásico de análisis filosófico. 
dese también las condiciones de verdad para esas proposiciones dadas en la sección 18.

El análisis contextualmente indexado del conocimiento de que $p$ se despliega tentativamente en el siguiente esquema, donde el suscrito ' $i$ ' es una letra esquemática que señala contextos epistémicos; sus substitutos adecuados son expresiones que denotan contextos epistémicos, cada una de las cuales, al ser suscrita a 'saber', representa un operador contextual que tiene el conocimiento genérico como operando y una especie de conocimiento (contextualmente identificada) como valor. Así, asumimos un contexto epistémico:

$$
C_{i}=\left\langle F_{i}, A_{i}, S_{i}, R_{i}, Q_{i}, P_{i}>\right.
$$

como se caracterizó anteriormente. Entonces:

$\mathrm{C}_{i} \mathrm{~K}$. En el tiempo $t \mathrm{X}$ sabe $_{i}$ que $p$ si y sólo si:

i) En el tiempo $t \mathrm{X}$ cree que $p$;

ii) $p$ está en $P_{i}$;

iii) Hay al menos una pregunta $q$ en $Q_{i}$ tal que:

1) $q \mathbf{S f}_{i} p$

2) si en $t \mathrm{X}$ considera $q$ y reflexiona, buscando en sus creencias una respuesta a $q, \mathrm{X}$ puede psicológicamente pensar con $\mathrm{fe}$, en $t^{\prime}$, que $q \mathrm{Sf}_{i} p$, donde el intervalo $\left(t, t^{\prime}\right)$ es un tiempo de computación y recuperación que concuerda con las restricciones que rigen la pregunta $q, y$

3) en $t \mathbf{X}$ cree que $q \mathbf{S f}_{i} p$;

iv) Hay circunstancias de verdad $Z$ al menos para algunos miembros de $P_{i}$, hay un número positivo $h$ y hay aspectos de anormalidad $r_{i}, \ldots, r_{h}$ en $R_{i}$ tales que:

1) $Z$ es un conjunto de circunstancias normales para esa $p$, excepto por incluir $r_{i}, \ldots, r_{h}$

2) $z$ se da, $y$

3) en $t \mathrm{X}$ cree que las circunstancias de verdad para esa $p$ son normales, excepto por incluir los aspectos $r_{i}, \ldots, r_{h}$;

v) Hay un subconjunto $s_{i}$ de $S_{i}$ tal que en $t \mathrm{X}$ tiene la propensión a hacer inferencias de acuerdo con los miembros de $s_{i}$ (no está excluido que pueda haber procesos de inferencia inconscientes o subconscientes y que algunos de ellos puedan ocurrir de acuerdo con los miembros de $s_{i}$ );

vi) Hay una conjunción $E_{i}$ de miembros de $F_{i}$ tal que:

1) Dados $Z$ y $s_{i}$, si $E_{i}$, entonces $p$;

2) Tanto $s_{i}$ como $E_{i}$ son verdaderos y

3) en $t \mathrm{X}$ cree que ambos, $E_{i}$ y, ceteris paribus [esto es, dada su actitud inferencial de creencia hacia $s_{i}$ y su creencia de que 
las circunstancias de verdad para esa $p$ son normales, excepto por $r_{i}, \ldots$, y $\left.r_{h}\right]$, si $E_{i}$, entonces $p .{ }^{37}$

\section{NOTAS ACERGA DEL ESQUEMA}

1. Las expresiones entre corchetes, en las cláusulas (v) y (vi), indican las anotaciones y referencias hechas por nosotros, o por cualquiera que use el esquema $C_{i} \mathrm{~K}$, para atribuir a alguien el conocimiento de que $p$ : no representan referencias ni partes del contenido del pensamiento de la persona $\mathrm{X}$, de la cual se dice que sabe.

2. Las ejemplificaciones particulares del esquema $C_{i} K$ pueden tener que formularse cuidadosamente debido a que acaso exijan cuasi-indicadores, ya sea para atribuir al conocedor $\mathrm{X}$ referencias demostrativas al tiempo (por usar $\mathbf{X}$ principalmente, en español, 'ahora'), al espacio (por usar 'aquí'), al propio ser (por usar 'yo'), etc. Véanse [6] y [7].

3. El esquema $C_{i} \mathrm{~K}$ se aplica al conocimiento matemático. Los estrictos estándares que prevalecen ahora, a fines del siglo $\mathrm{xx}$, para la prueba matemática son mucho más altos que los que prevalecían en el siglo xvir, los cuales eran a su vez más altos que los de dos siglos antes. Todas estas diferencias pertenecen a contextos epistémicos diferentes y están representadas genéricamente en las restricciones hechas a las preguntas de $Q_{i}$.

4. El esquema $C_{i} K$ tiene el siguiente patrón:

i) la condición de creencia;

ii) la condición de pertinencia;

iii) la condición del poder epistémico;

iv) la condición de la normalidad y anormalidad;

v) la condición del poder inferencial;

vi) la condición de la prueba en favor.

3. Escepticismo, conocimiento cientifico y otros temas. Creo que el esquema $\mathrm{C}_{\mathbf{i}} \mathrm{K}$ se conforma a todos los criterios de adecuación encontrados en la parte $\mathrm{I}$. Indudablemente el lector investigará si esto es o no así.

El esquema $\mathrm{C}_{i} \mathrm{~K}$ tiene la virtud de indicar a dónde pertenecen diferentes desarrollos. Cada uno de los distintos parámetros en un contexto epistémico da lugar a una rama de la epistemologia general. En particular, diferentes problemas de metodología de la ciencia tienen que ver con diferentes con-

37 Agradezco mucho a Peter French el haberme puesto en posición de terminar, al menos, este ensayo, aunque sea sólo la primera parte de una serie concebida y planeada en 1970 sobre el modelo de mi teoría del pensamiento práctico. Véase la nota 3 . La dilación fue realmente benéfica. Me permitió aprender de la rica literatura posterior a Gettier y referirme a ella. También me permitió utilizar los datos de Powers, así como incorporar su enfoque. 
juntos de restricciones, mismos que, al imponerse a las preguntas, determinan conjuntos unificados de objetivos epistémicos.

El problema del escepticismo, sus tipos y variantes, debe reabrirse. Obviamente, contextos epistémicos diversos dan lugar a distintos tipos de escepticismo. La mayoría de las formas radicales de escepticismo implican contextos con los parámetros más débiles que sea posible. Una versión radical del escepticismo, por ejemplo, funciona con el siguiente contexto epistémico:

$<\varnothing,\{X\}\{$ reglas de deducción $\}, \emptyset,\{$ todas las preguntas lógicamente posibles $\},\{$ todas las proposiciones $\}>$, donde $\emptyset$ es el conjunto vacío.

Claramente no es posible defender algunas formas extremas de escepticismo, mientras que otras son completamente defendibles, especialmente aquéllas, locales, acerca de algún pequeño grupo definido de proposiciones. Para determinar dónde trazar la línea entre escepticismos defendibles e indefendibles se requiere una investigación detallada. Parece que las cosas son más complejas de lo que comúnmente se piensa en relación al escepticismo. Una cosa es cierta: por más indefendible que pueda ser una forma de escepticismo, esto deberá mostrarlo la naturaleza del contexto epistémico implicado en esa forma de escepticismo. Es mejor evitar toda simple apelación a la falta de sentido del escepticismo. Ningún escéptico necesita usar 'saber' en una forma no ordinaria.

4. Colofón. Este ensayo ha culminado naturalmente en el esquema de análisis $\left(C_{\boldsymbol{i}} K\right)$. No tanto porque desarrolle un enfoque nuevo -inferencial, platónico-powersiano e indéxico- de la epistemologia básica, sino porque representa un esfuerzo metodológico sostenido por satisfacer a la vez las admoniciones de Sellars y de Wittgenstein usadas como epígrafes.*

HÉCTOR-NERI CASTAÑNEA

(Traducción del inglés por Sebastián Lamoyi)

\section{APÉNDICE}

1. Después de que el anterior ensayo había sido revisado para su publicación, Nuel Belnap me mostró que la jerarquía de preguntas descrita en la sección I.16 no incluye todas las preguntas a las que una proposición dada es una respuesta verdadera. Por ejemplo, la siguiente pregunta escapa a dicha jerarquia: ¿puede usted formular una proposición que sea un miembro del conjunto $S$ ? En general, las preguntas que se excluyen me parecen preguntas

- Cfr. Diánoia 1979, p. 126. 
que surgen de proposiciones que contienen a $P$ como un componente. Si esto es así, entonces la jerarquía descrita en la sección I.16 es sólo la jerarquia de preguntas propia o característica determinada por $P$. Me pregunto ahora si las llamadas restricciones a las preguntas en este ensayo pueden, al menos en algunos casos, considerarse partes de proposiciones que tienen como un componente una proposición dada en la que estamos interesados.

Sobre la teoría de las preguntas hay mucho más de lo que hemos tocado en nuestra discusión. Dado nuestro interés por el conocimiento y por el estado mental constitutivo del conocimiento, fue, sin embargo, crucial que no consideráramos a una pregunta como un conjunto de proposiciones, sino como un especial contenido de pensamiento que está en la relación $S f_{i}$ con sus respuestas. No estoy seguro de que la lógica de las preguntas que trata a las preguntas como conjuntos de proposiciones nos dé una explicación completamente satisfactoria de las actitudes interrogativas. Pero éste es un debate muy amplio que no podemos decidir aquí.

De cualquier manera, el análisis tentativo de la parte II permanece igual, creo, si se entiende que el parámetro $Q_{i}$ de preguntas incluye más preguntas de las que atendimos en nuestra discusión de la parte I. Véanse las ricas bibliografías de [1] y [2].

2. El 30 de octubre de 1979 George Nakhnikian me dio la siguiente cita:

Si por 'omnisciencia' queremos decir la aptitud para contestar con certeza toda pregunta concebible, incluyendo preguntas que conciernen al futuro... ([3], p. 121).

Así, en lugar de hablar del principio platónico-powersiano, como hice anteriormente, debí hablar del principio platónico-popperiano-powersiano.

\section{BIBLIOGRAFIA DEL APENDIGE}

[1] Belnap, Nuel, y Stell, Thomas B., Jr., The Logic of Questions and Answers (New Haven and London: Yale University Press, 1976).

[2] Hintikka, Jaakko, The Semantics of Questions and the Questions of Semantics (Amsterdam: North Holland Publishing Co., 1976).

[3] Popper, Karl, "Indeterminism in Quantum Physics and in Classical Physics", The British Journal for the Philosophy of Science 1 (1950-51).

\section{REFERENCIAS}

[1] Austin, John, "Other Minds", Aristotelian Society Supplementary Volume 20 (1946): 148-187.

[2] Alston, William, "Two Types of Foundationalism", The Journal of Philosophy 73 (1976): 165-185. 
[3] Carnap, Rudolf, Logical Foundations of Probability (Berkeley: University of California Press, 1949).

[4] Castañeda, Héctor-Neri, "Consciousness and Behavior: Their Basic Connections", en H. N. Castañeda, ed., Intentionality, Minds, and Perception (Detroit: Wayne State University Press, 1963).

[4a] Castañeda, Héctor-Neri, "Knowledge and Certainty", The Review of Metaphysics 18 (1965): 508-547.

[4b] Castañeda, Héctor-Neri, “There Are Command Sh-Inferences", Analysis 32 (1971): 13-19.

[5] Castañeda, Héctor-Neri, "On Knowing (or Believing) That One Knows (or Believes)", Synthese 21 (1970): 187-203.

[6] Castañeda, Héctor-Neri, "Indicators and Quasi-Indicators", American Philosophical Quarterly 4 (1967): 85-100.

[7] Castañeda, Héctor-Neri, "On the Philosophical Foundations of the Theory of Communication: Reference", Midwest Philosophical Studies 2 (1977): 165-186.

[8] Castañeda, Héctor-Neri, "The Causal and Epistemic Roles of Proper Names in Our Thinking of Particulars", en P. French, T. Wehling \& H. Wettstein, eds., Contemporary Perspectives in the Philosophy of Language (Minneapolis: University of Minnesota Press, 1979).

[9] Castañeda, Héctor-Neri, "Philosophical Method and Chisholm's Direct Awareness of Self", Grazer Philosophische Studien (de próxima aparición).

[10] Castañeda, Héctor-Neri, "Intentionality and Identity in Human Action and Philosophical Method", Noûs 13 (1979): 235-260.

[11] Castañeda, Héctor-Neri, Thinking and Doing: The Philosophical Foundations of Institutions (Dordrecht: D. Reidel, 1975).

[12] Castañeda, Héctor-Neri, "Perception, Belief, and the Structure of Physical Objects and of Consciousness", Synthese 35 (1977): 285-351.

[13] Chisholm, Roderick M., Person and Object: A Metaphysical Study (Chicago: Open Court, 1976).

[14] Chisholm, Roderick M., "Evidence as Justification", The Journal of Philosophy 58 (1961): 739-748.

[15] Chisholm, Roderick M., "Contrary-to-Duty Imperatives and Deontic Logic", Analysis 14 (1963): 33-36.

[16] Chisholm, Roderick M., Theory of Knowledge (Englewood Cliffs: Prentice-Hall, 1966).

[17] Chisholm, Roderick M., "Epistemic Statements and the Ethics of Belief", Philosophy and Phenomenological Research 16 (1956): 281-312.

[18] Chisholm, Roderick M., "Castañeda's Thinking and Doing", Noús 13 (1979): 000-000.

[19] Clark, Michael, "Knowledge and Grounds: A Comment on Mr. Gettier's Paper", Analysis 24 (1963): 46-48.

[20] Coder, David, "Thalberg's Defense of Justified True Belief", The Journal of Philosophy 67 (1970): 424-425.

[21] Coder, David, "Naturalizing the Gettier Argument", Philosophical Studies 26 (1974): 111-118.

[22] Cohen, L. Jonathan, The Implications of Induction (London: Methuen, 1970).

[23] Dretske, Fred, "Conclusive Reasons", Australasian Journal of Philosophy 49 (1971): 1-22. 
[24] Fichte, Johann Gottlieb, Science of Knowledge (New York: Appleton-CenturyCrofts, 1970; trad. al inglés por P. Heath y J. Lachs).

[25] Gettier, Edmund L., "Is Justified True Belief Knowledge?", Analysis 23 (1963): 121-123.

[26] Ginet, Carl, Knowledge, Perception, and Memory (Dordrecht: D. Reidel, 1975).

[27] Goldman, Alvin, "A Causal Theory of Knowing", The Journal of Philosophy 64 (1967): 355-372.

[28] Goldman, Alvin, "Discrimination and Perceptual Knowledge", The Journal of Philosophy 73 (1976): 771-791.

[29] Harman, Gilbert, Thought (Princeton: Princeton University Press, 1973).

[30] Heidegger, Martin, El ser y el tiempo (México: Fondo de Cultura Económica, $195 \mathrm{I}$; trad. por José Gaos).

[31] Heidelberg, Herbert, "Chisholm's Epistemic Principles", Noûs 19 (1979): 73-82.

[32] Hilpinen, Risto, "Knowing That One Knows and the Classical Definition of Knowledge", Synthese 21 (1970): 109-132.

[33] Hilpinen, Risto, "Knowledge and Justification", Ajatus 33 (1971): 7-39.

[34] Hintikka, Jaakko, Knowledge and Belief (Ithaca: Cornell University Press, 1962).

[35] Johnsen, Bredo, "Knowledge", Philosophical Studies 25 (1974): 273-282.

[36] Kant, Immanuel, Critique of Pure Reason. (London: Macmillan and Co. Ltd., 1933; trad. al inglés por Kemp Smith).

[37] Klein, Peter D., "A Proposed Definition of Propositional Knowledge", The Journal of Philosophy 68 (1971): 471-482.

[38] Klein, Peter D., "Knowledge, Causality, and Defeasibility", The Journal of Philosophy 73 (1976): 792-812.

[39] Kemeny, John G., "Fair Bets and Inductive Probabilities", The Journal of Symbolic Logic 20 (1955): 263-273.

[40] Lehrer, Keith, Knowledge (Oxford: Clarendon Press, 1974). Este libro supera a una docena más o menos de ensayos previos del propio Lehrer.

[41] Leibniz, Gottfried Wilhelm, "On the Method of Distinguishing Real from Imaginary Phenomena", en L. E. Loemker, ed., Gottfried Wilhelm Leibniz: Philosophical Papers and Letters (Dordrecht: D. Reidel, 1969).

[42] Loeb, Louis, "On a Heady Attempt to Befiend Causal Theories of Knowledge", Philosophical Studies 29 (1976): 331-336.

[43] Lucey, Kenneth, "Scales of Epistemic Appraisal", Philosophical Studies 25 (1974): 423-428.

[44] Pappas, George, "Knowledge and Reasons", Philosophical Studies 25 (1974): 423-428.

[45] Pappas, George y Swain, Marshall, "Some Conclusive Reasons Against 'Conclusive Reasons" ", The Australasian Journal of Philosophy 51 (1978): 72-76.

[46] Pastin, Mark, "Modest Foundationalism and Self-Warrant", en N. Rescher, ed., Studies in Epistemology (Oxford: Blackwells, 1975).

[47] Pastin, Mark, "Foundationalism Redux", The Journal of Philosophy 71 (1974): 709-710.

[48] Pastin, Mark, Review of Lehrer's Knowledge, Noûs 11 (1977): 431-437.

[49] Paxson, Thomas D., "Prof. Swain's Account of Knowledge", Philosophical Studies 25 (1974): 57-61.

[50] Powers, Lawrence, "Knowledge by Deduction", The Philosophical Review 87 (1978): 337-371. 
[51] Rescher, Nicholas, "Foundationalism, Coherentism and the Idea of Cognitive Systematization", The Journal of Philosophy 71 (1974): 695-708.

[52] Sartre, Jean-Paul, Being and Nothingness (New York: Philosophical Library, 1956; trad. al inglés por Hazel E. Barnes).

[53] Sellars, Wilfrid, "Meaning and Inference", Mind 62 (1953): 313-338.

[54] Sellars, Wilfrid, "Empiricism and the Philosophy of Mind", en H. Feigl \& $M$. Scriven, eds., Minnesota Studies in the Philosophy of Science, vol. 1 (Minneapolis University of Minnesota Press, 1956).

[55] Sellars, Wilfrid, "Phenomenalism", en H. N. Castañeda, ed., Intentionality, Minds, and Perception (Detroit: Wayne State University Press, 1967).

[56] Sellars, Wilfrid, "The Structure of Knowledge", en H.-N. Castañeda, ed. Action Knowledge and Reality: Studies in Honor of Wilfrid Sellars (Indianapolis: The Bobbs-Merrill Company, 1975).

[57] Shimony, Abner, "Coherence and the Axioms of Confirmation", The Journal of Symbolic Logic 20 (1955): 1-28.

[58] Skyrms, Brian, "The Explication of 'X knows that $p$ '", The Journal of Phylosophy 64 (1967): 333-389.

[59] Sosa, Ernest, "The Analysis of 'Knowledge that $p$ '", Analysis 25 (1964): 1-8.

[60] Sosa, Ernest, "Propositional Knowledge", Philosophical Studies 20 (1969): 33-43.

[61] Sosa, Ernest, "Two Conceptions of Knowledge", The Journal of Philosophy 67 (1970): 59-66.

[62] Sosa, Ennest, "The Concept of Knowledge. How Do You Know?", American Philosophical Quarterly 11 (1974): 113-122.

[63] Sosa, Ernest, "On Our Knowledge of Matters of Fact", Mind 83 (1974): 388-405.

[64] Sosa, Ernest, Review of Harman's thought, Noûs 11 (1977): 421-430.

[65] Sosa, Ernest, "The Foundations of Foundationalism", Noús (de próxima aparición).

[66] Swain, Marshall, "Knowledge, Causality, and Justification", The Journal of Philosophy 69 (1972): 291-300.

[67] Swain, Marshall, "Epistemic Defeasibility", American Philosophical Quarterly 11 (1974): 15-25.

[68] Thalberg, Irving, "In Defense of Justified True Belief", The Journal of Philosophy 66 (1969): 795-803.

[69] Tienson, John, "On Analyzing Knowledge", Philosophical Studies 25 (1974): 289-293.

[70] Tomberlin, James, "Knowing without Knowing that One Knows", Philosophia 2 (1972): 239-246.

[71] Tomberlin, James, Review of Carl Ginet's Knowledge, Perception and Memory, Nous (de próxima aparición).

[72] Villoro, Luis, "Conocer y saber", Critica No 10 (1970): 75-91.

[73] Wittgenstein, Ludwig, Philosophical Investigations (Oxford: Blackwells, 1952).

[74] Renault, Mary, Kinds Are Her Answers (Larchmont, New York: Queens House, 1978). 